\title{
Correlation of saponarin content with biosynthesis-related gene expression in hulled and hulless barley (Hordeum vulgare L.) cultivars
}

\author{
HanGyeol Lee $\cdot$ Jae-Hyeok Park A Mi Yoon $\cdot$ Young-Cheon Kim $\cdot$ Chul Soo Park $\cdot$ Ji Yeong Yang \\ So-Yeun Woo - Woo Duck Seo • Jeong Hwan Lee
}

Received: 6 January 2021 / Revised: 18 January 2021 / Accepted: 18 January 2021

(c) Korean Society for Plant Biotechnology

\begin{abstract}
Saponarin found in young barley sprouts has a variety of beneficial biological and pharmacological properties, including antioxidant, hypoglycemic, antimicrobial, and hepatoprotective activities. Our previous work demonstrated that saponarin content was correlated with the expression levels of three biosynthetic pathway genes [chalcone synthase (HvCHS1), chalcone isomerase $(H v C H I)$, and UDP-Glc:isovitexin 7-O-glucosyltransferase ( $H v O G T 1)]$ in young barley seedlings under various abiotic stress conditions. In this study, we investigated the saponarin content and expression levels of three saponarin biosynthetic pathway genes in hulled and hulless domestic barley cultivars. In the early developmental stages, some hulled barley cultivars (Kunalbori1 and Heukdahyang) had much higher saponarin contents than did the hulless barley cultivars. An RNA expression analysis showed that in most barley cultivars, decreased saponarin content correlated with reduced expression of $H v C H S 1$ and $H v C H I$, but not $H v O G T 1$. Heat map analysis revealed both specific increases in $\mathrm{HvCHSI}$ expression in certain hulled and hulless barley cultivars, as well as general changes that occurred during the different developmental stages of each barley cultivar. In summary, our results provide a molecular genetic basis for the metabolic engineering of barley plants to enhance their saponarin content.
\end{abstract}

H. G. Lee · J.-H. Park • A. M. Yoon • Y.-C. Kim • J. H. Lee $(\bowtie)$ Division of Life Sciences, Jeonbuk National University, 567 Baekjedaero, Deokjin-gu, Jeonju, Jeollabuk-do 54896, Republic of Korea e-mail: jhwanlee90@jbnu.ac.kr

C. S. Park

Department of Crop Science and Biotechnology, Jeonbuk National University, 567 Baekje-daero, Deokjin-gu, Jeonju, Jeollabuk-do 54896, Republic of Korea

H. G. Lee $\cdot$ J. Y. Yang $\cdot$ S.-Y. Woo $\cdot$ W. D. Seo $(\bowtie)$ Division of Crop Foundation, National Institute of Crop Science, Rural Development Administration, Jeollabuk-do 55365, Republic of Korea

e-mail: swd2002@korea.kr
Keywords Hulled and hulless barley cultivars, Chalcone isomerase, Chalcone synthase, Saponarin, UDP-Glc:isovitexin 7-O-glucosyltransferase

\section{Introduction}

Barley (Hordeum vulgare L.) is a major and historic cereal grain worldwide that has been cultivated for centuries as either animal feed, human food or malting substrate (Honsdorf et al. 2014). Over the past two decades, barley grains and leaves have ceased to be core livestock fodder and in turn their production has decreased. However, since secondary metabolites synthesized in young barley leaves contain the beneficial effect of potent antioxidants (Kamiyama and Shibamoto 2012), barley has begun to consider as a potential functional feed or food supplement for animals and humans, respectively.

Flavonoids are an important group of specialized plant metabolites and include the 10 major flavonoid classes: chalcones, aurones, flavanones, flavones, isoflavones, dihydroflavonols, flavonols, leucoanthocyanidins, anthocyanins and proanthocyanidins (Yonekura-Sakakibara et al. 2019). This structural diversity is a consequence of plant species adapting to specific ecological environments (Mouradov and Spangenberg 2014). Flavonoids contain a numerous of beneficial pharmacological effects (Seo et al. 2013). For instance, a diglycoside flavone (apigenin-6- $C$-glucosyl-7$O$-glucoside), also known as saponarin, when isolated from young barley sprouts, can promote anti-oxidant, hypoglycemic, anti-microbial and hepaprotective activities (Cushnie and Lamb 2005; Hertog et al. 1993; Mojzisova et al. 2006; Moses et al. 2014; Seo et al. 2014).

Flavonoid biosynthetic pathways have been thoroughly investigated in barley plants (Jende-Strid 1993; Peukert et al. 2013). To date, the sequences of chalcone synthase (CHS), 
phenylalanine ammonia-lyase (PAL), cinnamate 4-hydroxylase $(\mathrm{C} 4 \mathrm{H})$, flavanone 3-hydroxylase $(\mathrm{F} 3 \mathrm{H})$ and dihydroflavonol 4-reductase (DFR) genes have been identified in 16 barley genotypes. Single nucleotide polymorphism (SNP) markers for these respective genes have been found and developed. The genes encoding other flavonoid biosynthetic pathway enzymes, however, have yet to be discovered. The lack of available mutants in barley also hampers the identification of and functional study with new biosynthetic genes (Reuber et al. 1996).

Flavonoid biosynthetic pathways are evolutionarily conserved among plant species, and a variety of flavonoid biosynthetic genes have been well studied in plants (Lepiniec et al. 2006; Weisskopf et al. 2006). For instance, CHS catalyzing the first step in flavonoid synthesis is regulated at various levels of transcription and translation, and enzymatic activity (Block et al. 1990; Hartmann et al. 2005; Hartmann et al. 1998; Knogge and Weissenbock 1986). In addition, the levels of various genes involved in the flavonoid biosynthetic pathway are spatially and temporarily regulated in plant tissues (Hutzler et al. 1998; Schulz and Weissenbo"ck 1986; 1988). These findings suggest that the control of flavonoid production is more complex than expected.

We have recently reported on the relationship between the saponarin contents and expression levels of three saponarin biosynthesis genes [barley CHS1 (HvCHS1), barley chalcone isomerase $(\mathrm{HvCHI})$ and barley UDP-Glc: isovitexin 7-O-glucosyltransferase ( $H v O G T 1)$ ] in young barley seedlings during developmental stages and with several abiotic treatments (Lee et al. 2019). In this study, we analyzed the saponarin contents and saponarin biosynthetic gene expression in the same three biosynthesis genes for hulled and hulless domestic barley cultivars.

\section{Materials and Methods}

Plant materials and growth conditions

Barley seeds and cultivars were obtained from the National Institute of Crop Science (Jeonju, South Korea). These included 8 different barley seeds: Kunalbori1, Heukdahyang, 18CPB4, 18CPB10 and 18CPB 11 (hulled barley cultivars), as well as Nulichalssal, Hinchalssal and Saechalssal (hulless barley cultivars). 18CPB4, 18CPB10 and 18CPB 11 were not yet registered as new varieties (Yoon et al. 2019). According to our previous report (Lee et al. 2019), barley seeds soaked in water for $1 \mathrm{~d}$ were germinated in the darkness over $2 \mathrm{~d}$, transferred to soil, and then grown in a growth room $\left(23^{\circ} \mathrm{C}\right.$, 16-h photoperiod, light intensity of $120 \mathrm{mmol} \mathrm{m}^{-2} \mathrm{~s}^{-1}$ ). For saponarin content and biosynthesis gene expression analyses, young barley leaves were harvested at $3 \mathrm{~d}, 6 \mathrm{~d}$ or $9 \mathrm{~d}$. Afterwards, the samples were immediately frozen in liquid nitrogen for subsequent analysis. All experiments involved two or three biological replicates (independently harvested samples).

Saponarin content analysis of ultra-high performance liquid chromatography (UHPLC)

The saponarin contents in a variety of barley cultivars were analyzed in accordance with previous reports (Lee et al. 2019) (Seo et al. 2014). We prepared barley seedling extracts by incubating and shaking $0.5 \sim 1 \mathrm{~g}$ dried barley seedlings in $20 \mathrm{~mL} 80 \%$ methanol $(\mathrm{v} / \mathrm{v})$ at room temperature for $1 \mathrm{~d}$. UHPLC with a UV detector was implemented to measure the saponarin contents of the extracts. Three technical duplicates were performed for each of the two biological replicates (independently harvested samples). SPSS program for Windows (version 12.0, Seoul, Korea) was used for statistical analyses.

RNA expression analysis

Total RNA was extracted from the seedlings of hulled and hulless barely cultivars using Trizol reagent (Invitrogen, Carlsbad, CA, USA), and the quantity and quality of the resulting RNA were assessed using a Nanodrop ND-2000 spectrophotometer (Nanodrop Technologies, Waltham, MA, USA) and agarose gel electrophoresis, respectively. Complementary DNA (cDNA) was synthesized from $5 \mu \mathrm{g}$ of the total RNA, following the protocol of the ReverTra Ace qPCR RT Master Mix kit (Toyobo, Osaka, Japan), and real-time quantitative polymerase chain reaction (RT-qPCR) analysis was performed in 96-well plates using a CFX real-time system (Bio-Rad, Hercules, CA, USA), THUNDERBIRD SYBR qPCR mix (Toyobo). Gene-specific RTqPCR primers were designed for $H v C H S 1, H v C H I$ and HvOGT1 using QuantPrime (https://quantprime.mpimp-golm. mpg.de) (Arvidsson et al. 2008). $H v P P 2 A A 3$ was used as a stably expressed reference gene (Janska et al. 2013; Zhang et al. 2018), and three technical duplicates were performed for each of the two or three biological replicates. The primer sequences used for RNA expression analysis were previously reported (Lee et al. 2019). To examine the relative abundance of the transcripts, the data were analyzed using Bio-Rad CFX Manager (Bio-Rad). A heat map was presented using heatmapper (http://www2.heatmapper.ca). SPSS program 
for Windows (version 12.0, Seoul, Korea) was used for statistical analyses.

\section{Results and Discussion}

Saponarin contents in hulled and hulless barley cultivars

Since previous reports have shown that saponarin accumulates substantially during the primary barley leaf developmental stage (Lee et al. 2019; Reuber et al. 1996), we examined saponarin contents in hulled and hulless domestic barley cultivars grown at $23{ }^{\circ} \mathrm{C}$ under long-day (LD) conditions for $3 \mathrm{~d}$ and $9 \mathrm{~d}$. We used 8 different barley cultivars including Kunalbori1, Heukdahyang, 18CPB4, 18CPB10 and 18CPB 11 (hulled barley cultivars), and Nulichalssal, Hinchalssal and Saechalssal (hulless barley cultivars) and compared their saponarin contents. UHPLC analysis revealed that saponarin contents accumulated significantly at $3 \mathrm{~d}$ and were reduced at $9 \mathrm{~d}$ in all barley cultivars tested in this study (Fig. 1). This result was consistent with our previous report (Lee et al. 2019). High levels of saponarin content, however, were observed at the earliest stages of the third day in Kunalboril and Heukdahyang (hulled barley cultivars) compared with others [18CPB4, 18CPB10 and 18CPB 11 (hulled barley cultivars) and Nulichalssal,

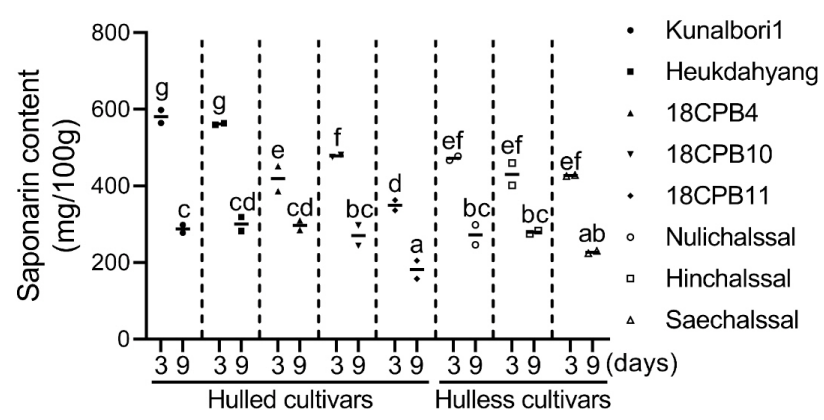

Fig. 1 Saponarin content in young seedlings of hulled and hulless barley cultivars. Barley seedlings grown at $23^{\circ} \mathrm{C}$ under long-day (LD) conditions were harvested at $3 \mathrm{~d}$ and $9 \mathrm{~d}$, and the accumulation of saponarin was determined by ultra-high performance liquid chromatography (UHPLC) analysis. Different letters indicate statistically significant groups, as determined by the Duncan method for multiple comparisons $(P<0.05)$. Comparisons were performed within cultivars and days

Hinchalssal and Saechalssal (hulless barley cultivars)] at the same time. The differences in saponarin content levels between hulled and hulless barley cultivars may be due to variances in parental lines used for barley breeding (http:// seed.go.kr). For instance, Kunalbori1 and Heukdahyang originated from Kunalbori and Kunalboril, respectively. Nulichalssal, Hinchalssal and Saechalssal originated from Yonezawa as their common parental line.
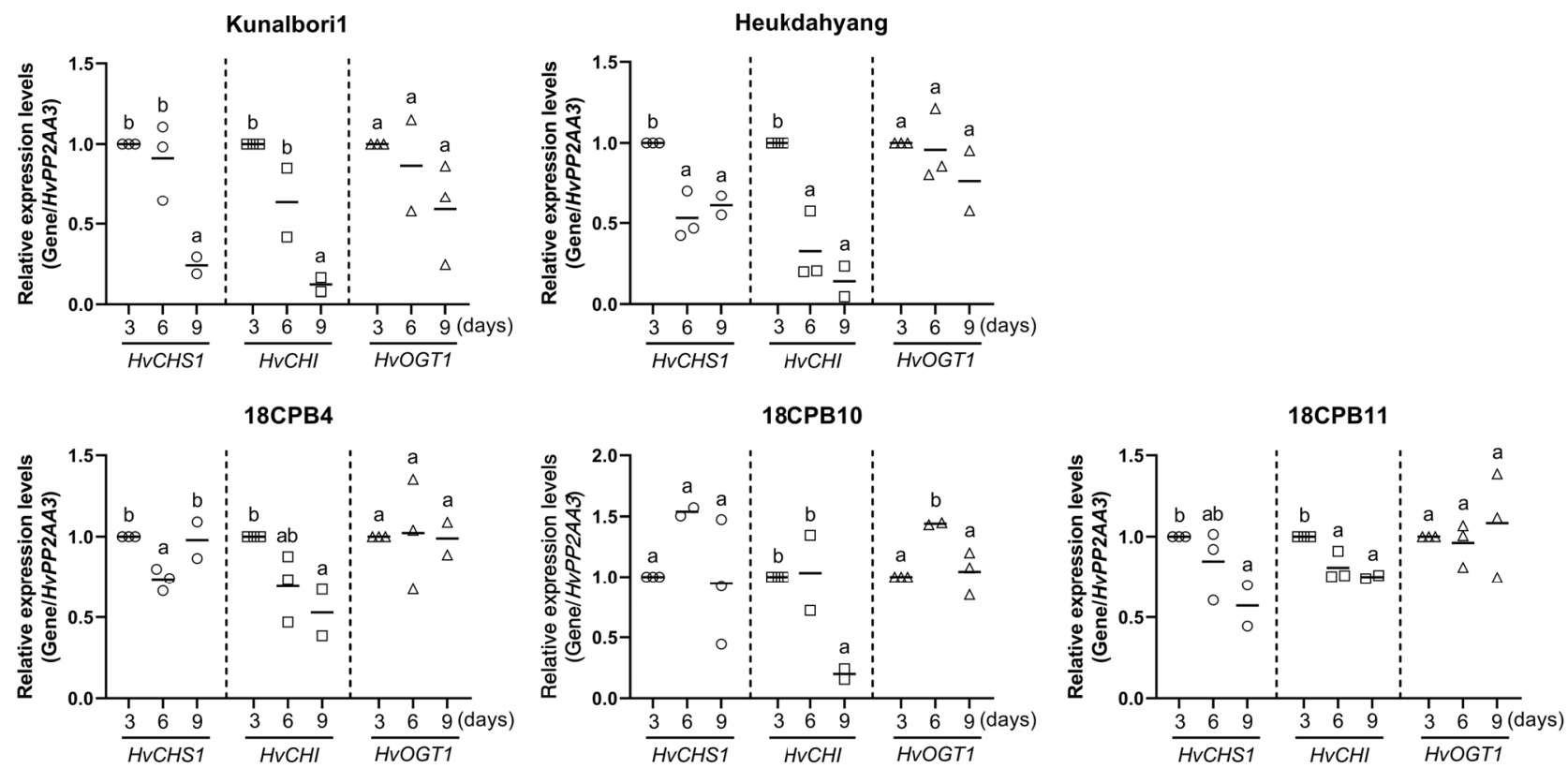

Fig. 2 Expression levels of $H v C H S 1, H v C H I$, and $H v O G T 1$ in young seedlings of hulled barley cultivars. Barley seedlings grown at $23^{\circ} \mathrm{C}$ under long-day (LD) conditions were harvested at $3 \mathrm{~d}, 6 \mathrm{~d}$, and $9 \mathrm{~d}$. Real-time quantitative PCR (RT-qPCR) was used to analyze the expression levels of $H v C H S 1, H v C H I$, and $H v O G T 1$ in five barley cultivars. At $3 \mathrm{~d}$, each gene's expression level was set to 1. Statistical analysis was performed using the Duncan method for multiple comparisons. Comparisons were performed within cultivars and days. Different letters indicate statistically significant groups $(P<0.05)$ 

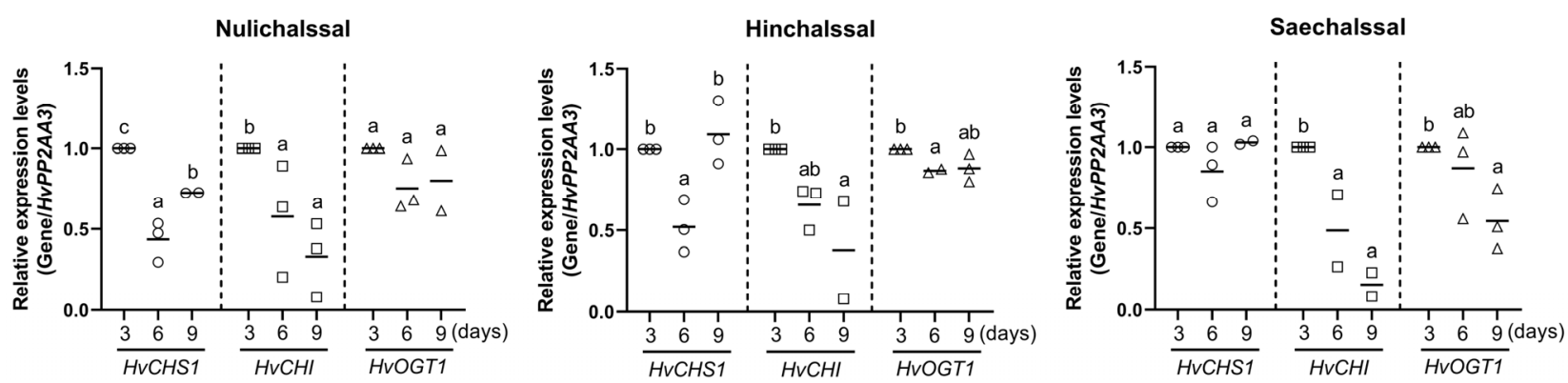

Fig. 3 Expression levels of $H v C H S 1, H v C H I$, and $H v O G T 1$ in young seedlings of hulless barley cultivars. Barley seedlings grown at $23^{\circ} \mathrm{C}$ under long-day (LD) conditions were harvested at $3 \mathrm{~d}, 6 \mathrm{~d}$, and $9 \mathrm{~d}$. Real-time quantitative PCR (RT-qPCR) was used to analyze the expression levels of $H v C H S 1, H v C H I$, and $H v O G T 1$ in three barley cultivars. At $3 \mathrm{~d}$, each gene's expression level was set to 1 . Statistical analysis was performed using the Duncan method for multiple comparisons. Comparisons were performed within cultivars and days. Different letters indicate statistically significant groups $(P<0.05)$

Expression of three saponarin biosynthetic genes in hulled and hulless barley cultivars

Saponarin contents accumulated substantially at $3 \mathrm{~d}$ and were dramatically reduced at $9 \mathrm{~d}$ in all barley cultivars (Fig. 1). In order to find whether the changes in the saponarin contents of the barley cultivars were correlated with the expression patterns of saponarin biosynthesis genes, we investigated the expression of $\mathrm{HvCHSl}, \mathrm{HvCHI}$ and $\mathrm{HvOGTI}$ in barley seedlings. Barley plants were grown at $23^{\circ} \mathrm{C}$ under LD conditions and harvested after $3 \mathrm{~d}, 6 \mathrm{~d}$ and 9 d. In Kunalbori1, Heukdahyang, 18CPB4, 18CPB10 and $18 \mathrm{CPB} 11$ (hulled barley cultivars), expression analysis showed that $H v C H S 1$ and $H v C H I$ expression decreased significantly at $6 \mathrm{~d}$ or $9 \mathrm{~d}$, whereas $H v O G T 1$ expression was unaltered (Fig. 2). In Nulichalssal, Hinchalssal and Saechalssal (hulless barley cultivars), similar expression patterns were observed (Fig. 3). These results are consistent with previous results that $C H S$ and $C H I$ expression in plants are controlled in a temporal manner (Hutzler et al. 1998; Schulz and Weissenbo"ck 1986; 1988). Moreover, heat map analysis revealed that changes in HvCHS1 expression appeared among certain barley cultivars, or during developmental stages in the same barley cultivar (Fig. 4). For instance, higher expression levels of $\mathrm{HvCHS1}$ at $3 \mathrm{~d}$ were found in Kunalboril and Heukdahyang with a high accumulation of saponarin contents on the same day, compared with the expression levels in other barley cultivars tested. In addition, a gradual decrease in the expression of $H v C H S 1$ from $3 \mathrm{~d}$ to $9 \mathrm{~d}$ was observed in most barley cultivars, which indicated the correlation between saponarin contents and $H v C H S 1$ expression at same developmental stage. These results suggest that the decreased expression levels of $H v C H S 1$ and $H v C H I$, at an early point within the saponarin biosynthetic pathway, may result in

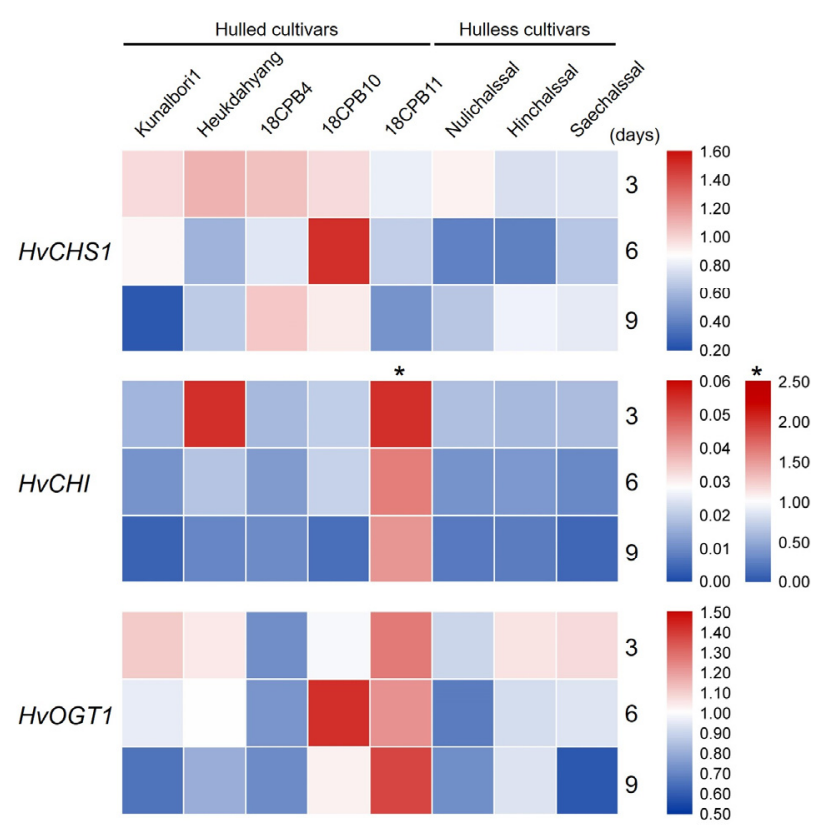

Fig. 4 Heat map analysis of $H v C H S 1, H v C H I$, and $H v O G T 1$ expression in hulled and hulless barley cultivars. Absolute expression patterns of the three saponarin biosynthetic genes $H v C H S 1, H v C H I$, and HvOGT1 are presented as a heat map, using the genes' average expression levels over two or three biological replicates. The color scale (shown at right) indicates each gene's expression pattern in a given cultivar: higher expression level is indicated by red color, whereas lower expression level is indicated by blue color. Note that the expression of $\mathrm{HvCHI}$ was much higher in the cultivar 18CBP11 than in the other cultivars; in turn, this gene/cultivar combination has a different color scale, indicated by an asterisk

the decreased accumulation of saponarin content at a later developmental stage in barley cultivars.

\section{Conclusion}

Saponarin has recently been shown to have various medicinal 
effects on human health (Cushnie and Lamb 2005; Hertog et al. 1993; Mojzisova et al. 2006; Moses et al. 2014; Seo et al. 2014). However, we do not fully understand the correlation between the saponarin contents and expression of saponarin biosynthetic genes in different barley cultivars. In this work, we investigated the saponarin content and the expression levels of three saponarin biosynthetic genes in hulled and hulless domestic barley cultivars. Saponarin contents in the early developmental stage of hulled barley cultivars were much higher than those in hulless barley cultivars (Fig. 1). The changes in saponarin content were correlated with altered expression levels of $\mathrm{HvCHS1}$ and $H v C H I$, but not $H v O G T 1$ (Figs. 2, 3 and 4). It is likely that the sequence variation(s) of cis-acting elements within their promoter regions may cause the changes in the expression patterns of $H v C H S 1$ and $H v C H I$, thereby resulting in changing levels of saponarin content in hulled and hulless barley cultivars. Alternatively, the sequence changes of various transcription factors affecting the expression of saponarin biosynthetic genes may be involved in the modulation of saponarin contents. This notion is supported by the observation that the MBW protein complex containing MYB, bHLH and WD40, repeat factors usually regulate the groups of flavonoid biosynthetic genes (Davies and Schwinn 2003; Niu et al. 2010; Ravaglia et al. 2013). Thus, further research on which transcription factors affect the expression levels of $\mathrm{HvCHSI}$ and $\mathrm{HvCHI}$ and control the levels of saponarin content would provide a better understanding of the saponarin biosynthesis pathway in barley plants.

\section{Acknowledgements}

This work was supported by grants from the New Breeding Technologies Development Program of the Rural Development Administration (Project No. PJ01532503 to J.H.Lee) and the "Cooperative Research Program for Agriculture Science \& Technology Development of Rural Development Administration (Project title: 'Analysis and standardization of functional compounds, change in acidification of Korean sweets', Project No. PJ01528303 to W.D.Seo)", Republic of Korea. We wish to express our gratitude towards Prof. Seong-Woo Cho (Gyeongnam National University of Science and Technology), Hye Rim Shin, Young Ho Won and Ji Woo Kim for their assistance.

\section{References}

Arvidsson S, Kwasniewski M, Riano-Pachon DM, Mueller-Roeber
B (2008) QuantPrime--a flexible tool for reliable highthroughput primer design for quantitative PCR. BMC Bioinformatics 9:465

Block A, Dangl JL, Hahlbrock K, Schulze-Lefert P (1990) Functional borders, genetic fine structure, and distance requirements of cis elements mediating light responsiveness of the parsley chalcone synthase promoter. Proc Natl Acad Sci USA 87:5387-5391

Cushnie TP, Lamb AJ (2005) Antimicrobial activity of flavonoids. Int J Antimicrob Agents 26:343-356

Davies KM, Schwinn KE (2003) Transcriptional regulation of secondary metabolism. Funct Plant Biol 30:913-925

Hartmann U, Sagasser M, Mehrtens F, Stracke R, Weisshaar B (2005) Differential combinatorial interactions of cis-acting elements recognized by R2R3-MYB, BZIP, and BHLH factors control light-responsive and tissue-specific activation of phenylpropanoid biosynthesis genes. Plant Mol Biol 57:155-171

Hartmann U, Valentine WJ, Christie JM, Hays J, Jenkins GI, Weisshaar B (1998) Identification of UV/blue light-response elements in the Arabidopsis thaliana chalcone synthase promoter using a homologous protoplast transient expression system. Plant Mol Biol 36:741-754

Hertog MG, Feskens EJ, Hollman PC, Katan MB, Kromhout D (1993) Dietary antioxidant flavonoids and risk of coronary heart disease: the Zutphen Elderly Study. Lancet 342:1007-1011

Honsdorf N, March TJ, Berger B, Tester M, Pillen K (2014) High-throughput phenotyping to detect drought tolerance QTL in wild barley introgression lines. PLoS One 9:e97047

Hutzler P, Fischbach R, Heller W, Jungblut T, Reuber S, Schmitz R, Veit M, Weissenbo“ck G, Schnitzler J (1998) Tissue localization of phenolic compounds in plants by confocal laser scanning microscopy. J Exp Bot 49:953-965

Janska A, Hodek J, Svoboda P, Zamecnik J, Prasil IT, Vlasakova E, Milella L, Ovesna J (2013) The choice of reference gene set for assessing gene expression in barley (Hordeum vulgare L.) under low temperature and drought stress. Mol Genet Genomics 288:639-649

Jende-Strid B (1993) Genetic control of flavonoid biosynthesis in barley. Hereditas 119:187-204

Kamiyama M, Shibamoto T (2012) Flavonoids with Potent Antioxidant Activity Found in Young Green Barley Leaves. J Agric Food Chem 60:6260-6267

Knogge W, Weissenbock G(1986) Tissue-distribution of secondary phenolic biosynthesis in developing primary leaves of Avena sativa L. Planta 167:196-205

Lee H, Woo S-Y, Ra J-E, Lee K-S, Seo WD, Lee JH (2019) Saponarin content and biosynthesis-related gene expression in young barley (Hordeum vulgare L.) seedlings. J Plant Biotechnol 46:247-254

Lepiniec L, Debeaujon I, Routaboul JM, Baudry A, Pourcel L, Nesi N, Caboche M (2006) Genetics and biochemistry of seed flavonoids. Annu Rev Plant Biol 57:405-430

Mojzisova G, Mirossay L, Kucerova D, Kyselovic J, Mirossay A, Mojzis J (2006) Protective effect of selected flavonoids on in 
vitro daunorubicin-induced cardiotoxicity. Phytother Res 20:110-114

Moses T, Papadopoulou KK, Osbourn A (2014) Metabolic and functional diversity of saponins, biosynthetic intermediates and semi-synthetic derivatives. Crit Rev Biochem Mol Biol 49:439-462

Mouradov A, Spangenberg G (2014) Flavonoids: a metabolic network mediating plants adaptation to their real estate. Front Plant Sci 5:620

Niu SS, Xu CJ, Zhang WS, Zhang B, Li X, Lin-Wang K, Ferguson IB, Allan AC, Chen KS (2010) Coordinated regulation of anthocyanin biosynthesis in Chinese bayberry (Myrica rubra) fruit by a R2R3 MYB transcription factor. Planta 231:887-899

Peukert M, Weise S, Roder MS, Matthies IE (2013) Development of SNP markers for genes of the phenylpropanoid pathway and their association to kernel and malting traits in barley. BMC Genetics 14:16

Ravaglia D, Espley RV, Henry-Kirk RA, Andreotti C, Ziosi V, Hellens RP, Costa G, Allan AC (2013) Transcriptional regulation of flavonoid biosynthesis in nectarine (Prunus persica) by a set of R2R3 MYB transcription factors. BMC Plant Biol 13:68

Reuber S, Bornman JF, Weissenbo“ck G (1996) A flavonoid mutant of barley (Hordeum vulgare L.) exhibits increased sensitivity to UV-B radiation in the primary leaf. Plant Cell Environ 19:593-601

Schulz M, Weissenbo“ck G (1986) Isolation and separation of epidermal and mesophyll protoplasts from rye primary leaves: tissue specific characteristics of secondary phenolic product accumulation. Z Naturforsch [C] 41:22-27

Schulz M, Weissenbo“ck G (1988) Dynamics of the tissue-specific metabolism of luteolin glucuronides in the mesophyll of rye primary leaves (Secale cereale). Z Naturforsch [C] 43:187-193

Seo KH, Park MJ, Ra JE, Han SI, Nam MH, Kim JH, Lee JH, Seo
WD (2014) Saponarin from barley sprouts inhibits NF-kappaB and MAPK on LPS-induced RAW 264.7 cells. Food Funct 5:3005-3013

Seo WD, Yuk HJ, Curtis-Long MJ, Jang KC, Lee JH, Han SI, Kang HW, Nam MH, Lee SJ, Lee JH, Park KH (2013) Effect of the growth stage and cultivar on policosanol profiles of barley sprouts and their adenosine 5'-monophosphate-activated protein kinase activation. J Agric Food Chem 61:1117-1123

Weisskopf L, Abou-Mansour E, Fromin N, Tomasi N, Santelia D, Edelkott I, Neumann G, Aragno M, Tabacchi R, Martinoia E (2006) White lupin has developed a complex strategy to limit microbial degradation of secreted citrate required for phosphate acquisition. Plant Cell Environ 29:919-927

Wu X, Zhang S, Liu X, Shang J, Zhang A, Zhu Z, Zha D (2020) Chalcone synthase (CHS) family members analysis from eggplant (Solanum melongena L.) in the flavonoid biosynthetic pathway and expression patterns in response to heat stress. PLoS One 15:e0226537

Yonekura-Sakakibara K, Higashi Y, Nakabayashi R (2019) The Origin and Evolution of Plant Flavonoid Metabolism. Front Plant Sci 10:943

Yoon Y-M, Kim K-H, Kang T-G, Kang S-W, Kang H-J, -S. KC, Kim Y-K, Park CS, Cho S-W (2019) Evaluation of Starch Properties of Korean Barley (Hordeum vulgare L.) Cultivars. Korean J Breed Sci 51:73-85

Zhang L, Zhang Q, Jiang Y, Li Y, Zhang H, Li R (2018) Reference genes identification for normalization of qPCR under multiple stresses in Hordeum brevisubulatum. Plant Methods $14: 110$

Zu QL, Qu YY, Ni ZY, Zheng K, Chen Q, Chen QJ (2019) The Chalcone Isomerase Family in Cotton: Whole-Genome Bioinformatic and Expression Analyses of the Gossypium barbadense L. Response to Fusarium Wilt Infection. Genes (Basel) 10 